\title{
Autonomic neuropathy and coeliac disease
}

\author{
C H Gibbons, R Freeman
}

J Neurol Neurosurg Psychiatry 2005;76:579-581. doi: 10.1136/jnnp.2004.047480

Coeliac disease is associated with numerous neurological manifestations including cerebellar ataxia, myelopathy, myopathy, and peripheral neuropathy. This report describes four patients who presented subacutely with presyncope and postural nausea. All four patients had biopsy proven coeliac disease with dysautonomia present on autonomic evaluation. These four patients comprised $2.4 \%$ of patients referred for autonomic testing in one year. Thus the frequency of coeliac disease is similar to that reported in idiopathic peripheral neuropathy.

C oeliac disease (gluten sensitive enteropathy) is the commonest manifestation of gluten sensitivity, however, diverse manifestations may accompany the disorder. ${ }^{12}$ Several recent reports have drawn attention to the association between gluten sensitivity and elevated antigliadin antibodies and neurological disorders. Neurological manifestations associated with gluten sensitivity include cerebellar ataxia, myelopathy, myopathy, and peripheral neuropathy. ${ }^{12}$ Although the prevalence of antigliadin antibodies in patients with idiopathic peripheral neuropathy may be as high as $40 \%,{ }^{1}$ a recent study suggested that the prevalence of biopsy proven coeliac disease in a referral population of idiopathic peripheral neuropathy patients is approximately $2.5 \% .^{2}$

Dysautonomia is seen in disorders of the central and peripheral nervous systems. In many patients, however, no underlying cause is found. ${ }^{3}$ In one series, patients with known coeliac disease had subclinical abnormalities of autonomic function when tested but no autonomic symptoms other than the gastrointestinal symptoms characteristic of coeliac disease. ${ }^{4}$ We report four patients with biopsy proven coeliac disease and symptomatic, laboratory confirmed dysautonomia from a group of 164 patients referred for autonomic testing.

\section{PATIENTS AND METHODS}

We retrospectively reviewed the history, physical examination, and autonomic testing of all patients referred to the Autonomic Function Laboratory for suspected autonomic dysfunction during a one year period. Four patients were identified among 164 to have coeliac disease and autonomic dysfunction. The patients' details are summarised in table 1.

Other identifiable causes of dysautonomia were excluded. One patient with positive IgA antigliadin antibodies without confirmatory biopsy was excluded. A sixth patient with elevated antigliadin antibodies and a positive biopsy was excluded due to a history of diabetes as a possible cause of dysautonomia.

All four patients initially presented to their general practitioner with nausea, were diagnosed as having coeliac disease by IgA antigliadin antibodies, and had characteristic findings on small bowel biopsy. All had a diagnosis of biopsy confirmed coeliac disease prior to neurological evaluation.
Initiation of a gluten free diet did not improve the nausea. Patients were referred for autonomic evaluation due to symptoms of nausea and lightheadedness on average one year (range 8-14 months) from the diagnosis of coeliac disease.

All patients described nausea that occurred while sitting or standing but was absent while supine. All gave a history of syncopal or presyncopal episodes pre-dating the diagnosis of coeliac disease. Other autonomic symptoms included palpitations and lightheadedness. None of the patients experienced traditional symptoms of coeliac disease such as diarrhoea, and only one (patient 4) experienced constipation.

All patients had normal cognitive, cranial nerve, motor, reflex, coordination, sensory, and gait examinations. Autonomic testing included expiratory to inspiratory heart rate variability, Valsalva manoeuvre with blood pressure and heart rate analysis, tilt table testing to $60^{\circ}$ for 45 minutes, and five minute stand. We compared the results to age related normative values. ${ }^{5}$ The results of the autonomic tests are shown in table 1. Abnormalities in parasympathetic and sympathetic function were present. The heart rate response to the Valsalva manoeuvre, a measure of cardiac parasympathetic nervous system function, was abnormal in three of four patients. In addition, abnormal measures of sympathetic nervous system function were present. The blood pressure response to the Valsalva manoeuvre was abnormal in all patients (fig 1). Two patients had orthostatic hypotension on tilt table testing and active standing (a fall greater than $20 \mathrm{~mm} \mathrm{Hg}$ systolic or $10 \mathrm{~mm} \mathrm{Hg}$ diastolic blood pressures). ${ }^{6}$ The other two patients had a postural tachycardia syndrome (defined as rise in heart rate of 30 beats per minute or more without a significant fall in blood pressure). ${ }^{7}$

The patients did not notice a change in symptoms while following a gluten restricted diet, however, they all indicated poor compliance. In two of the four patients repeated testing for antigliadin antibodies demonstrated a reduction, but not an absence, of antibodies. Treatment with volume expansion and $\alpha$-adrenoreceptor agonist therapy gave some relief of symptoms but none of the patients returned to baseline level of functioning.

\section{DISCUSSION}

We report four patients with antigliadin antibodies, coeliac enteropathy, and dysautonomia. Although the association between neurological disease and gluten sensitivity has been reported frequently, ${ }^{2}$ given the high percentage of antigliadin antibodies in the general population $(6-12 \%)$ the aetiological significance of this association is uncertain in most patients. ${ }^{8}$ Still less is known about the potential mechanisms whereby gluten sensitivity might result in neurotoxicity. Nutritional factors and other disorders, such as diabetes and Sjögren's syndrome, that are associated with coeliac disease and may cause neurological illness were excluded in our patients. It is speculated that in genetically predisposed individuals antigliadin antibodies or other associated antibodies may cause nerve injury. ${ }^{20}$ Although antigliadin antibodies have been associated with ataxia and 
Table 1 Characteristics and autonomic test results of patients

\begin{tabular}{|c|c|c|c|c|}
\hline & \multicolumn{4}{|l|}{ Patient } \\
\hline & 1 & 2 & 3 & 4 \\
\hline Age/Sex & $38 / \mathrm{M}$ & $78 / \mathrm{F}$ & $54 / F$ & $63 / \mathrm{F}$ \\
\hline Symptoms & $\begin{array}{l}\text { Postural nausea; } \\
\text { lightheadedness; } \\
\text { presyncope }\end{array}$ & $\begin{array}{l}\text { Postural nausea; } \\
\text { lightheadedness; } \\
\text { syncope }\end{array}$ & $\begin{array}{l}\text { Postural nausea; } \\
\text { dizziness; } \\
\text { lightheadedness; } \\
\text { syncope }\end{array}$ & $\begin{array}{l}\text { Postural nausea; } \\
\text { lightheadedness } \\
\text { weakness; fatigue; } \\
\text { syncope }\end{array}$ \\
\hline Baseline heart rate (beats per minute) & 75 & 66 & 72 & 85 \\
\hline Baseline blood pressure $(\mathrm{mm} \mathrm{Hg})$ & $121 / 64$ & $142 / 74$ & $128 / 66$ & $180 / 92$ \\
\hline Heart rate variation with respiration (average max-min) & $23(>12 \mathrm{nl})$ & $10(>7 \mathrm{nl})$ & $11(>9 \mathrm{nl})$ & $6^{*}(>7 \mathrm{nl})$ \\
\hline Heart rate response to Valsalva manoeuvre & $1.33^{*}(>1.5 \mathrm{nl})$ & $1.44(>1.39 \mathrm{nl})$ & $1.19^{*}(>1.47 \mathrm{nl})$ & $1.07^{*}(>1.39 \mathrm{nl})$ \\
\hline Highest heart rate response to tilt & $112^{*}$ & 86 & $104^{*}$ & 108 \\
\hline Lowest blood pressure response to tilt & $112 / 60$ & $105 / 52^{*}$ & $122 / 72$ & $114 / 62^{*}$ \\
\hline Highest heart rate response to five minute stand & 106 & 84 & 116 & 110 \\
\hline Lowest blood pressure response to five minute stand & $116 / 68$ & $115 / 68^{*}$ & $132 / 70$ & $128 / 64^{*}$ \\
\hline
\end{tabular}

peripheral neuropathy, the relation between these antibodies and dysautonomia is not established. In a single report of patients with coeliac disease and oesophageal dysmotility, subclinical abnormalities of cardiovascular reflexes were present in $19 \%(5 / 27)$ of patients. ${ }^{4}$

In our patients, nausea, which was postural in nature, was the primary symptom for referral. Other reported autonomic symptoms included lightheadedness, palpitations, fatigue, presyncope, and syncope. Autonomic test results revealed abnormalities in sympathetic and parasympathetic nervous system functions. These symptoms and test findings are not unique to coeliac disease patients and can be seen in other patients with dysautonomia. Our patients did not improve on a gluten restricted diet, an experience reported by other investigators. ${ }^{2}$ There are several possible explanations for this. Firstly, the patients were not closely monitored, and all admitted to dietary indiscretion. The two patients with repeated antibody testing had titres that, although reduced, remained elevated, suggestive of continued dietary intake of gluten. Secondly, it is possible that structural nerve damage, once present, is not responsive to dietary measures.'

Patients with gluten sensitivity and symptomatic dysautonomia have not been previously described. At our laboratory, $2.4 \%$ of 164 patients referred for autonomic evaluation had idiopathic dysautonomia and biopsy proven coeliac disease, a frequency similar to that reported in patients with idiopathic
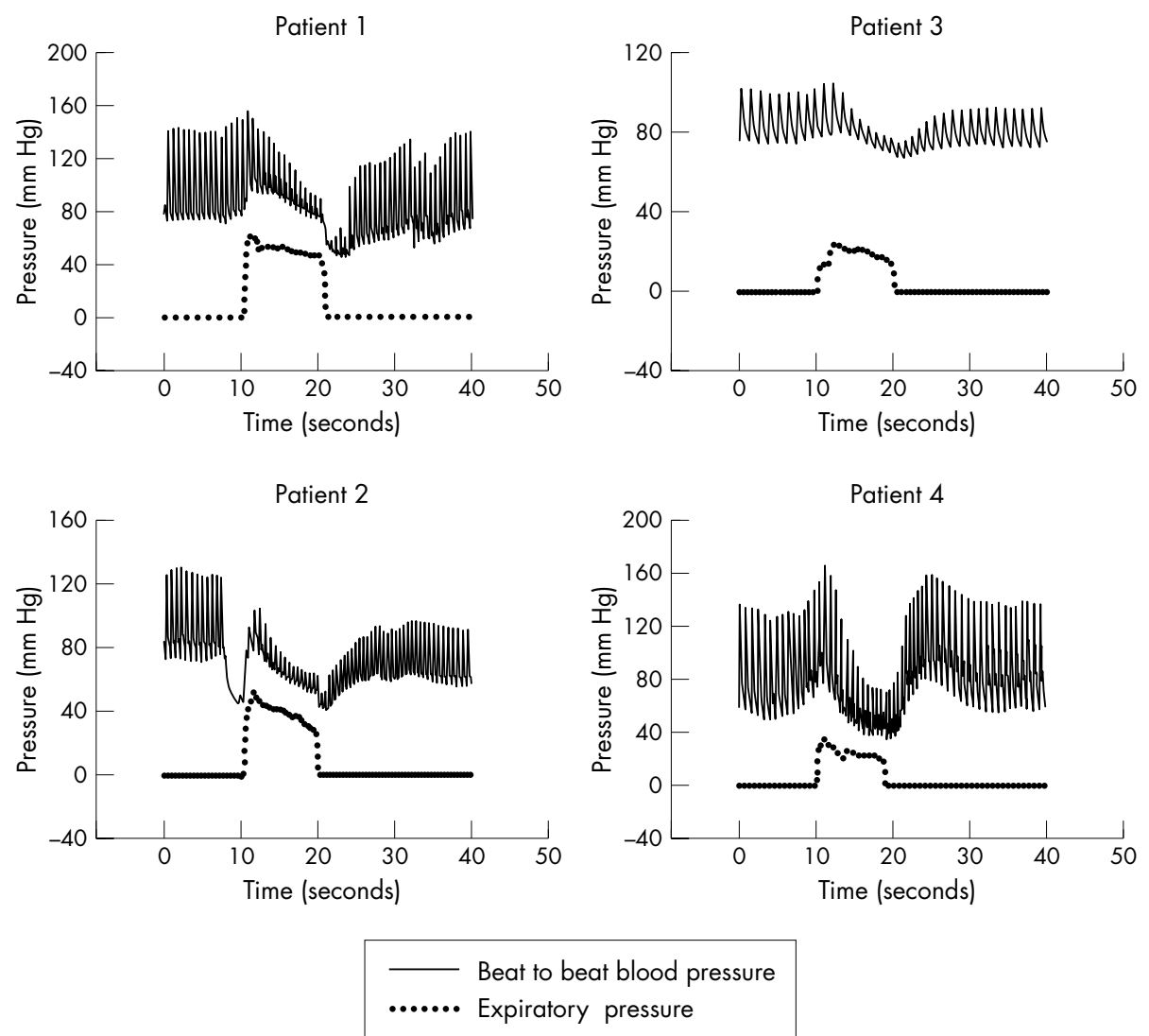

Figure 1 The response of each patient to a Valsalva manoeuvre is shown. In each graph, the beat to beat blood pressure is shown on the top and the expiratory pressure on the bottom. All patients had reduced pulse pressure during phase II, and no late phase II recovery of blood pressure. Patient 2 showed an abnormal phase I of the Valsalva. Only patient 4 showed a phase IV overshoot. 
peripheral neuropathy. ${ }^{2}$ If corroborated by additional studies, this report could suggest that screening for coeliac disease should be considered in selected patients with autonomic neuropathy of uncertain aetiology. A prospective study evaluating patients presenting with idiopathic dysautonomia for coeliac disease is warranted.

\section{Authors' affiliations}

C H Gibbons, R Freeman, Department of Neurology, Beth Israel Deaconess Medical Center, Harvard Medical School, Boston, MA, USA Competing interests: none declared

Correspondence to: Dr R Freeman, Autonomic and Peripheral Nerve Laboratory, Department of Neurology, Beth Israel Deaconess Medical Center, 1 Deaconess Road, Boston, MA 02215, USA; rfreeman@bidmc. harvard.edu

Received 11 June 2004

Revised version received 26 July 2004

Accepted 14 August 2004

\section{REFERENCES}

1 Hadjivassiliou M, Grunewald RA, Davies-Jones GA. Gluten sensitivity as a neurological illness. J Neurol Neurosurg Psychiatry 2002;72:560-3.

2 Chin RL, Sander HW, Brannagan TH, et al. Celiac neuropathy. Neurology 2003;60:1581-5

3 Mathias CJ, Bannister R. Autonomic Failure, 4th edn. Oxford: Oxford University Press, 1999

4 Usai $P$, Usai SP, Lai $M$, et al. Autonomic dysfunction and upper digestive functional disorders in untreated adult coeliac disease. Eur $J$ Clin Invest 1997; 27:1009-15.

5 Low PA. Laboratory evaluation of autonomic function. In: Low PA, ed. Clinical autonomic disorders: evaluation and management. Philadelphia: LippincottRaven, 1997:179-208.

6 Position paper: Orthostatic hypotension, multiple system atrophy (the Shy Drager syndrome) and pure autonomic failure. J Auton Nerv Syst 1996;58:123-4.

7 Low PA, Opfer-Gehrking TL, Textor SC, et al. Postural tachycardia syndrome (POTS). Neurology, 1994;(suppl).

8 Cross AH, Golumbek PT. Neurologic manifestations of celiac disease: proven, or just a gut feeling? Neurology 2003;60:1566-8.

9 Hadjivassiliou M, Davies-Jones GA, Sanders DS, et al. Dietary treatment of gluten ataxia. J Neurol Neurosurg Psychiatry 2003;74:1221-4.

10 Shill HA, Alaedini A, Latov N, et al. Anti-ganglioside antibodies in idiopathic and hereditary cerebellar degeneration. Neurology 2003;60:1672-3. 